\title{
Spontaneous Autoimmune Dacryoadenitis in Aged CD25KO Mice
}

\author{
Ehsan Rahimy, ${ }^{*}$ John D. Pitcher III, ${ }^{*}$ \\ Solherny B. Pangelinan, ${ }^{*}$ Wei Chen, ${ }^{* \dagger}$ \\ William J. Farley, ${ }^{*}$ Jerry Y. Niederkorn, ${ }^{\neq}$ \\ Michael E. Stern, ${ }^{\S}$ De-Quan Li, ${ }^{*}$ \\ Stephen C. Pflugfelder, ${ }^{*}$ and Cintia S. De Paiva* \\ From the Ocular Surface Center," the Department of \\ Ophthalmology, Cullen Eye Institute, Baylor College of Medicine, \\ Houston, Texas; the School of Ophthalmology and Optometry, ${ }^{\dagger}$ \\ Eye Hospital, Wenzhou Medical College, Wenzhou, Zhejiang, \\ China; the Department of Ophthalmology, ${ }^{\ddagger}$ University of Texas \\ Southwestern Medical Center, Dallas, Texas; and the Department \\ of Biological Sciences, ${ }^{\S}$ Allergan Inc., Irvine, California
}

To investigate time-related immunopathological changes in the lacrimal glands (LGs) of CD25KO mice, we examined LGs of C57BL/6 (wild-type) and CD25KO mice at 8,12 , and 16 weeks of age. $T$ cell infiltration was quantified by flow cytometry, and gland function by tear peroxidase activity and epidermal growth factor mRNA expression. T helper (Th)-1, -2 and -17associated cytokine expression was evaluated by realtime PCR. Epithelial apoptosis was assessed by terminal deoxynucleotidyl transferase dUTP nick-end labeling assay and activated caspase- 3 staining. Eightweek-old CD25KO mice demonstrated significantly increased numbers of CD4 and CD8 $\mathrm{T}$ cells infiltrating the LGs. This peaked at 12 weeks of age. No peroxidase secretion was detected, and epidermal growth factor mRNA expression was barely detected in CD25KO mice. Ductal epithelial apoptosis was noted in CD25KO mice. Young CD25KO LGs had higher Th17- (interleukin [IL]-23R, transforming growth factor$\beta 1, I L-17 A, C C$ chemokine attractant ligand-20) and Th-1-associated cytokine transcripts (interferon- $\gamma$, Tbet, IL-12, IL-2, IL-18) than young wild-type LGs. There was also a significant time-related decrease in IL-17A and CC chemokine attractant ligand-20 in CD25KO LGs. Taken together, autoimmune LG infiltration with loss of LG function was observed in CD25KO mice as early as 8 weeks of age. Time-related switch from Th-17 to Th-1 inflammation was noted in CD25KO mice. (Am J Pathol 2010, 177:744-753; DOI: 10.2353/ajpath.2010.091116)
Aging has been shown to decrease lacrimal gland (LG) function, with a resultant increase in the incidence of dry eye, affecting 15 to $25 \%$ of people over the age of 65 years. ${ }^{1}$ Age-related structural changes observed in both murine and postmortem human LG include: acinar atrophy, periductal fibrosis, interlobular duct dilatation, accumulation of lipofuscin granules, and an infiltration of mast cells followed by lymphocytes expressing pro-inflammatory cytokines. ${ }^{2-4}$ An ensuing state of chronic inflammation is believed to contribute to the LG secretory dysfunction that often develops in the elderly.

Dry eye is a common complaint of patients visiting eye care specialists. Patients typically present with irritative symptoms such as burning, dryness, and foreign body sensation. Signs may include decreased tear meniscus, increased tear film debris, corneal irregularity secondary to punctate epithelial erosions, and in more severe cases, corneal filaments and mucous plaques. Its impact on quality of life can be severe, impairing the ability to perform simple daily activities such as driving, reading, and computer use. ${ }^{5}$ Dry eye affects functional vision, with a significant decrease in low contrast acuity vision. ${ }^{6}$ Among dry eye patients, $11 \%$ are estimated to have Sjögren's syndrome (SS), a chronic autoimmune disease primarily affecting women (>90\%) in their fifth and sixth

Supported by National Institutes of Health grants EY11915 (S.C.P.); Fight for Sight grants-in-aid (C.S.D.P.); Hartford Foundation (C.S.D.P.); Research to Prevent Blindness, Oshman Foundation; William Stamps Farish Fund; Hamill Foundation and an unrestricted grant from Allergan Inc. The sponsor agencies had no involvement on the study design; data collection, analysis and interpretation of data; in the writing of the report; and in the decision to submit the paper for publication.

Accepted for publication April 6, 2010.

Presented in part as abstract at the annual meeting of the Association for Research in Vision and Ophthalmology, April 27-May 1, 2009, Fort Lauderdale, Florida.

Disclosures: M.E.S. is an employee of Allergen, Inc. J.Y.N. has stock options in a for-profit company. None of the other authors declare any relevant financial relationships.

Supplemental material for this article can be found on http://ajp. amjpathol.org.

Address reprint requests to Cintia S. De Paiva, M.D., Cullen Eye Institute, Baylor College of Medicine, 6565 Fannin Street, NC 205- Houston, TX 77030. E-mail: cintiadp@bcm.tmc.edu. 
decades of life. ${ }^{7}$ Characterized by a lymphocytic infiltration of the lacrimal and salivary glands, SS clinically manifests with symptoms of dry eye (lacrimal keratoconjunctivitis) and dry mouth (xerostomia). ${ }^{7}$

The mechanisms underlying lacrimal gland dysfunction in SS are not fully understood, but epithelial apoptosis is thought to have a role in the secretory impairment. Studies in SS patients have demonstrated the presence of apoptosis-regulating proteins in the exocrine tissue such as the pro-apoptotic molecules Fas antigen (Apo1/CD95), Fas ligand (FasL/CD95L), and Bax. ${ }^{8-10}$ Additional studies in animals with SS-like disease suggest $T$ cells are able to induce glandular apoptosis by one of three mechanisms: 1) an interaction between Fas antigen on the epithelial surface and Fas ligand, expressed by the lymphocytes; 2) release of perforins and granzyme $\mathrm{B}$ by cytotoxtic T cells; and 3) production of pro-apoptotic cytokines such as tumor necrosis factor- $\alpha$ and interferon (IFN)- $\gamma^{8,10}$

The recently identified T-helper (Th)-17 population of $\mathrm{CD}^{+} \mathrm{T}$ cells has challenged the Th- $1 / \mathrm{Th}-2$ paradigm of $\mathrm{T}$ cell development established over 20 years ago. Th- 1 cells contribute to host defense of viral, fungal, and intracellular bacteria infections, and are characterized by the production of IFN- $\gamma$. Th-2 cells are known to participate in allergic disease, and the eradication of gut parasites and helminthes. This pathway is characterized by the production of interleukin (IL)-4, IL-5, and IL-13 cytokines. The remaining fates for a naïve $T$ cell point toward mutually exclusive development of either Th-17 or regulatory T cell based on the local cytokine milieu. Mice studies have found that transforming growth factor $\beta$ (TGF- $\beta$ ) in the presence of either IL-6 or IL-21 is sufficient to drive Th-17 differentiation, ${ }^{11-13}$ with its specific transcription factor, retinoid-related orphan receptor- $\gamma \tau$, whereas, the expression of just TGF- $\beta$ will induce a regulatory T cell development with its specific transcription factor, forkhead box protein 3 (Foxp3). ${ }^{11-13}$ Th-17 committed cells can produce IL-17A, IL-17F, IL-21, IL-22, and CC chemokine attractant ligand-20 (CCL20). ${ }^{14,15} \mathrm{IL}-17 \mathrm{~A}$, the signature cytokine of this pathway, functions as a pro-inflammatory mediator by stimulating the local production of IL-1, IL-6, IL-8, and tumor necrosis factor- $\alpha$ by epithelial cells and fibroblasts, and can further potentiate tissue destruction by inducing the production of matrix metalloproteinases (MMPs). ${ }^{16,17}$

The Th-17 pathway has been implicated in the development of autoimmunity in humans and animal models, such as inflammatory bowel disease, experimental autoimmune encephalomyelitis, and collagen induced arthritis. ${ }^{18-20}$ IL-17A has been found to be elevated in the synovium of rheumatoid arthritis patients, ${ }^{21}$ sera of patients with systemic lupus erythematosus, ${ }^{22}$ active brain lesions of multiple sclerosis patients, ${ }^{23}$ and vitreous of patients with uveitis. ${ }^{24} \mathrm{IL}-17 \mathrm{~A}$ has also been implicated in the acute corneal barrier dysfunction in an experimental dry eye model, and increased IL-17A mRNA transcript levels were identified in conjunctiva of dry eye patients. ${ }^{25}$ Furthermore, IL-17A expression has been found in salivary gland ductal epithelial cells of SS patients and nonobese diabetic (NOD) mice. ${ }^{26,27}$
Numerous animal models of SS have been proposed in an attempt to elucidate the pathogenesis of the disease. Some of the more studied murine models include MRL/ Ipr, NOD, and NZB/NZW. As the role of the CD4+ CD25+Foxp3+ regulatory T cell (Treg) in maintaining peripheral tolerance and controlling organ-specific autoimmunity has expanded, ${ }^{28,29}$ an IL-2R $\alpha^{-1-}$ (CD25KO) mouse model emerged as a model of SS given that it develops a multi-organ inflammatory syndrome involving the exocrine glands, gastrointestinal tract, and a profound antibody-mediated hemolytic anemia. ${ }^{30,31}$ Regulatory $T$ cells normally express a high-affinity form of the IL-2 receptor, composed of the IL-2R $\beta$ (CD122) and $\gamma$ chains complexed with the $\alpha$ subunit (CD25). Binding of IL-2 to its receptor in Treg cells induces expression of Foxp3, and promotes cell survival. ${ }^{32}$ Additionally, IL-2 has been shown to antagonize differentiation of the Th-17 pathway. ${ }^{32}$ Thus, absence of the high-affinity $\alpha$ subunit could skew the murine naivve cells toward Th-17 and away from regulatory $T$ cell differentiation, promoting inflammation and autoimmunity.

The purpose of this study was to investigate timerelated changes in function and immunopathology in the LG of IL-2R $\alpha^{-1-}$ (CD25KO) mice, with emphasis on the T cell-associated cytokine expression. A better understanding of the pathological mechanism behind SS at the molecular level will assist in the development of targeted therapeutics to control the disease process, rather than symptomatic treatments currently available.

\section{Materials and Methods}

This research protocol was approved by the Baylor College of Medicine Center for Comparative Medicine, and it conformed to the standards in the Association for Research in Vision and Ophthalmology Statement for the Use of Animals in Ophthalmic and Vision Research.

Heterozygous breeder pairs of CD25+/- mice in a

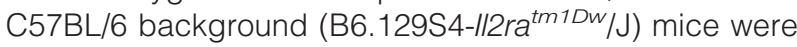
purchased from Jackson Laboratories (Bar Harbor, Maine) for establishing breeder colonies. They were housed in our vivarium in a pathogen-free environment. The genotype of CD25KO mice was confirmed according to the Jackson Labs' protocol. C57BL/6 wild-type mice were purchased from Jackson Laboratories.

Mice of both genders were used at 8,12 , and 16 weeks of age. Fifty-two animals per time point $(8,12$, and 16 weeks) per strain (CD25KO and wild-type) were used: 5 mice for histological sections, 20 mice for tear collection, and 20 mice for gene analysis, and 6 animals for flow cytometry analysis.

\section{Histology}

Extraorbital lacrimal glands were surgically excised, fixed in $10 \%$ formalin, paraffin embedded, and $8-\mu \mathrm{m}$ sections were cut. Sections were stained with H\&E for evaluation of morphology. The area of T cell infiltration was circumscribed in digital images of H\&E-stained sections, using 
NIS Elements (Nikon, Melville, NY), and the percentage of infiltration calculated using the total area of the LG.

For immunohistochemistry, terminal deoxyribonucleotidyl transferase-mediated d-UTP nick-end labeling (TUNEL) and immunofluorescent assays, extraorbital lacrimal glands from each strain/time point $(n=5)$ were excised, embedded in optimal cutting temperature compound (VWR, Suwanee, GA), and flash frozen in liquid nitrogen. Sagittal 8- $\mu \mathrm{m}$ sections were cut with a cryostat (HM 500; Micron, Waldorf, Germany) and placed on glass slides that were stored at $-80^{\circ} \mathrm{C}$.

\section{Tear Peroxidase Assay}

Tear peroxidase activity was used as a measure of LG function. ${ }^{33}$ Tear fluid washings were collected from 20 animals/group, in two independent experiments, using a previously reported method. ${ }^{25}$ One sample consisted of tear washings from both eyes of two mice pooled $(4 \mu \mathrm{l})$ in PBS $+0.1 \%$ bovine serum albumin $(6 \mu \mathrm{l})$ and stored at $-80^{\circ} \mathrm{C}$ until the assay was performed. Tear peroxidase measurement was performed using an Amplex Red Peroxidase Kit, according to the manufacturer's instructions (Invitrogen-Molecular Probes, Eugene, OR). There were a total of 10 samples from each strain per time point.

\section{Immunohistochemistry}

Immunohistochemistry was performed to detect and count the cells in the LG that stained positively for CD4 (clone H129.9, $10 \mu \mathrm{g} / \mathrm{ml}$ ) and CD8 $\alpha$ (clone 53e6.7, 3.125 $\mu \mathrm{g} / \mathrm{ml}$ ), [from BD Bioscience, San Diego, CA]. Cryosections were stained with the above mentioned primary antibodies and appropriate biotinylated secondary antibodies (all from BD Pharmingen) and a Vectastain Elite $A B C$ kit using NovaRed reagents (Vector, Burlingame, CA) as previously described. ${ }^{9}$ Secondary antibody alone and appropriate anti-mouse isotype (BD Bioscience) controls were also performed. Three sections from each animal/group/time point were examined and photographed with a microscope equipped with a digital camera (Eclipse E400 with a DS-Fi1; Nikon).

\section{Flow Cytometry Analysis of Infiltrating Cells}

Single-cell suspensions of lacrimal glands of C57BL/6 and CD25KO at 8,12, and 16 weeks of age $(n=3)$ were prepared by treatment of minced tissue fragments with $0.1 \%$ collagenase D (60 minutes at $37^{\circ} \mathrm{C}$, InvitrogenGibco, Carlsbad, California) and sequentially filtered. Single-cell suspensions were stained with antiCD16/32 (to block Fc receptors, BD Pharmigen, San Diego, CA, $4^{\circ} \mathrm{C}, 10$ minutes), followed by cell surface staining with anti-CD4 (GK1.5; BD Pharmigen), or antiCD8 (clone 53-6.7, BD Pharmingen). Negative controls were stained with fluorescein isothiocyanate-isotype antibody (BD Pharmigen). Cells were resuspended in violet dye (live/dead cell fixable staining, Invitrogen-Molecular Probes, Carlsbad, CA) and washed. Cells were then resuspended in fixation-permeabilization solution (Cytofix)
Cytoperm; BD Pharmingen) and stored at $4^{\circ} \mathrm{C}$ until the next day when the analysis was performed. A BD LSRII Benchtop cytometer was used for flow cytometry and data were analyzed using BD Diva Software (BD Pharmigen).

\section{Immunofluorescent Staining and Laser Scanning Confocal Microscopy}

Cryosections stained for activated caspase $3(5 \mu \mathrm{g} / \mathrm{ml}$, BD Bioscience) were developed using goat anti-rabbit Alexa-Fluor 488 conjugated IgG antibody as previously described. ${ }^{34}$ Negative controls performed at the same time consisted of sections incubated with an isotype antibody (BD Bioscience) or sections with the primary antibody omitted.

Digital images $(512 \times 512$ pixels $)$ of cryosections were captured with a laser-scanning confocal microscope (LSM 510; Zeiss with krypton-argon and He-Ne laser; Carl Zeiss Meditec, Ltd. Thornwood, NY) with 488-excitation and 543-nm emission filters, LP505 and LP560, respectively. They were acquired with a $40 / 1.3 \times$ oil-immersion objective.

\section{TUNEL Assay}

The TUNEL assay was performed using a kit (ApopTag; Intergen Co., Purchase, NY). ${ }^{35}$ Cryosections were fixed in $1 \%$ paraformaldehyde, and permeabilized with 2:1 ethanol:acetic acid solution. The samples were incubated with TdT enzyme and 11 -digoxigenin dUTP at $37^{\circ} \mathrm{C}$ for 4 hours. After quenching the reaction, samples were blocked with blocking solution and incubated with antidigoxigenin fluorescein isothiocyanate-conjugated antibody for 60 minutes at room temperature.

\section{RNA Isolation and Real-Time PCR}

Total LG RNA from CD25KO and wild-type mice at each time point $(8,12$, and 16 weeks) was extracted using an acid guanidium thiocyanate-phenol-chloroform method as previously described. ${ }^{34}$ Four samples per strain were used, and one sample consisted of pooled LG from five mice per time point. Samples were treated with DNase to prevent genomic DNA contamination according to the manufacturer's instructions (Qiagen, Valencia, CA).

First-strand cDNA was synthesized from $1 \mu \mathrm{g}$ of total RNA using random hexamers and M-MuLV reverse transcriptase (Ready-To-Go You-Prime First-Strand Beads; GE Health care, Inc., Piscataway, NJ), as previously described. ${ }^{34}$ Real-time PCR was performed using gene expression assay primers and MGB probes specific for murine target mRNAs (Table 1). The glyceraldehyde-3phosphate dehydrogenase gene was used as an endogenous reference for each reaction. The results of quantitative PCR were analyzed by the comparative $\mathrm{C}_{\mathrm{T}}$ method where target change $=2^{\Delta \Delta C T}$ (User Bulletin, No. 2, P/N 4303859; $\mathrm{ABI}$ ). The cycle threshold (CT) was determined using the primary (fluorescent) signal as the cycle at 
Table 1. Classification of Target mRNAs Investigated by Real-Time PCR

\begin{tabular}{cc}
\hline Target mRNA & Assay IDs \\
\hline Th-17 pathway & \\
GAPDH & Mm99999915 \\
MMP-9 & Mm00442991 \\
IL-6 & Mm00446490 \\
TGF- $\beta 1$ & Mm00441724 \\
TGF- $\beta 2$ & Mm00436952 \\
IL-15 & Mm00434210 \\
IL-17A & Mm00439619 \\
IL-17R & Mm00434214 \\
IL-17F & Mm00521423 \\
IL-23 & Mm00518984 \\
IL-23R & Mm00519942 \\
ROR $\gamma$ t & Mm00441139 \\
IL-21 & Mm00517640 \\
IL-22 & Mm00444241 \\
CCL20 & Mm00444228 \\
STAT3 & Mm00456961 \\
Th-1 pathway & \\
IFN- $\gamma$ & Mm00801778 \\
IL-2 & Mm00434256 \\
IL-12 & Mm00434165 \\
IL-12RB1 & Mm00434189 \\
IL-18 & Mm00434225 \\
IL-18R & Mm00515180 \\
T-bet & Mm00450960 \\
Th-2 pathway & IL-4 \\
IL-13 & Mm00445259 \\
GATA-3 & Mm00434204 \\
\hline
\end{tabular}

which the signal crossed a user-defined threshold. The results were normalized by the CT value of glyceraldehyde-3-phosphate dehydrogenase and the mean CT of relative mRNA level in the C57BL/6 group at 8 weeks of age was used as the calibrator.

\section{Statistical Analysis}

One-way analysis of variance (analysis of variance) with Tukey's post hoc testing was used for statistical comparisons of tear cytokines and gene expression profiles in cornea and conjunctiva. $P \leq 0.05$ was considered statistically significant. These tests were performed using GraphPad Prism 5.0 software (GraphPad Software Incorporation, San Diego, CA).

\section{Results}

\section{Spontaneous Dacryoadenitis in the CD25KO Mice}

The LG undergoes an array of changes in its structural organization with aging. ${ }^{2-4}$ To determine the effect of time-related changes, extra-orbital LG of wild-type and CD25KO mice strains of both genders were excised and prepared for histological examination. During surgical excision, CD25KO LG were noted to be enlarged, red, and inflamed at 8 and 12 weeks of age, while at 16 weeks the LGs were small and atrophic compared with wild-type LGs. At the cellular level, young C57BL/6 wild-type LG
A

A
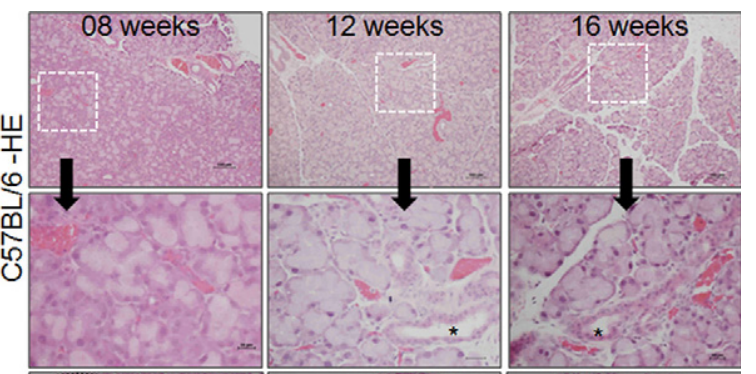

C

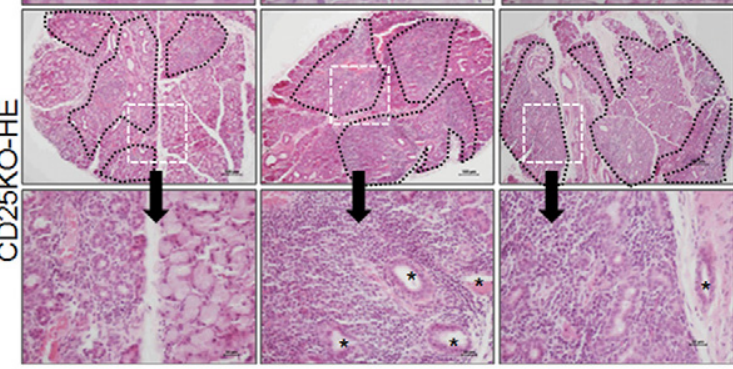

E
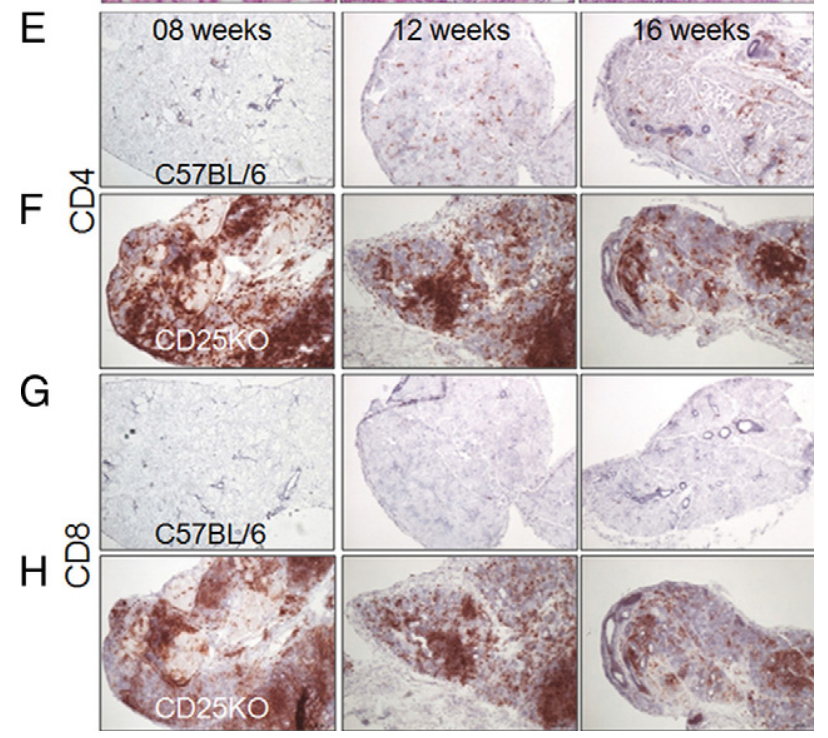

Figure 1. A-D: Representative images of H\&E-stained lacrimal gland (LG) sections of $\mathrm{C} 57 \mathrm{BL} / 6$ wild-type $(\mathbf{A}, \mathbf{B})$ and $\operatorname{CD} 25 \mathrm{KO}(\mathbf{C}, \mathbf{D})$ mice at 8,12 , and 16 weeks of age. $\mathbf{A}$ and $\mathbf{C}$ : Images of original magnification $\times 10$. $\mathbf{B}$ and $\mathbf{D}$ : Higher magnification of white square demarcated area in (A). Note lymphocytic infiltration (circumscribed by black dotted lines) at all time points in CD25KO mice. Openings of epithelial ducts are marked by asterisks. $\mathbf{E}-\mathbf{H}$ : Immunohistochemistry for CD4 $(\mathbf{E}-\mathbf{F})$ and $\mathrm{CD} 8(\mathbf{G}, \mathbf{H})$ positive T cells in the LG of C57BL/6 wild-type and $\mathrm{CD} 25 \mathrm{KO}$ mice at 8,12 , and 16 weeks of age.

exhibited normal gland architecture, with plump acini (Figure 1, A and B), while young CD25KO LG showed acinar atrophy, acinar fibrosis, periductal fibrosis, and intense lymphocytic infiltration occupying up to $70 \%$ of the total area of the gland (Figure 1, C and D). These findings in the CD25KO mice further worsened at 12 weeks. At 16 weeks of age, CD25KO LG continued to show lymphocytic infiltration, but greater disarrangement of the gland was noted, with extensive acinar loss and atrophy of the entire gland. LG sections stained for CD4 and CD8 demonstrated a significantly higher density of these $\mathrm{T}$ cells in CD25KO mice (Figure 1, $\mathrm{F}$ and $\mathrm{H}$ ) when compared with C57BL/6 wild-type mice (Figure 1, E and $G)$ at all ages. To further quantify the infiltrating immune cells, we performed flow cytometry analysis in LG of both 
A

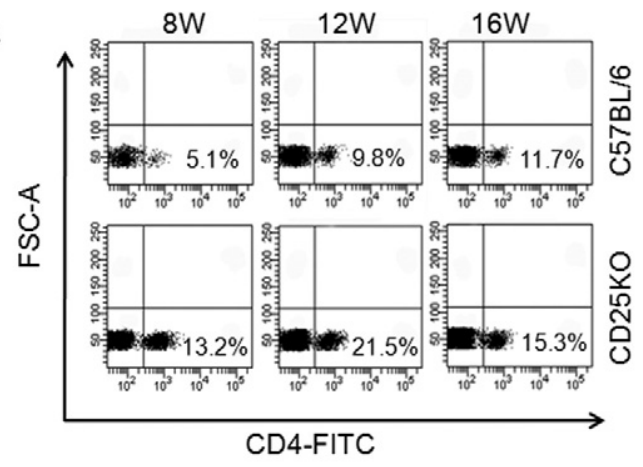

B

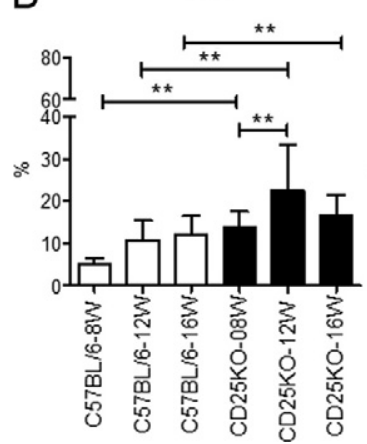

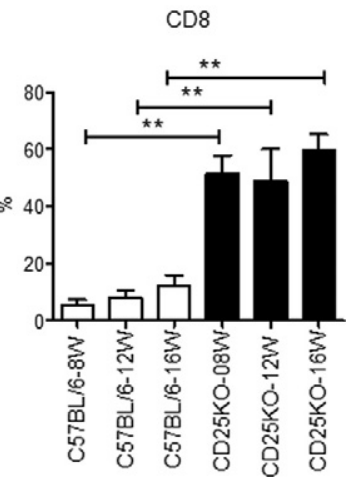

Figure 2. A: Representative flow cytometry analysis of freshly cells stained for CD 4 from LG of CD25KO and C57BL/ 6 mice at 8, 12, and 16 weeks of age ( $8 \mathrm{~W}, 12 \mathrm{~W}$, and $16 \mathrm{~W}$, respectively). Lymphocytes were gated based on characteristic light-scatter properties; single lymphocytes were gated based on forward scatter height versus forward scatter area (FSC-A). Numbers in the quadrants indicate the percentage of cells. B: Mean \pm SD of three independent flow cytometry (FACS) experiments of CD4 and CD8+ T cells in LG of $\mathrm{CD} 25 \mathrm{KO}$ and $\mathrm{C} 57 \mathrm{BL} / 6$ mice at 8,12 , and 16 weeks of age $(8 \mathrm{~W}, 12 \mathrm{~W}$, and $16 \mathrm{~W}$, respectively). ${ }^{* *} P<0.01$.

strains, at all time points, for CD4 and CD8. Our results, presented in Figure 2, A and $\mathrm{B}$ demonstrated that CD25KO mice had significantly higher percentage of CD4 and CD8+ T cells at all ages, compared with C57BL/6 wild-type mice. CD8 $+\mathrm{T}$ cells were the dominant cell type. There were no gender-related differences in the amount of infiltrating cells in either strain (data not shown).

These results suggest that time-related structural changes occurred in LG of CD25KO as early as 8 weeks. The ocular surface pathology of these mice were investigated in another study. ${ }^{36}$

\section{Lack of Gender Differences in CD25KO LG Pathology}

Sjogren's syndrome disproportionally affects women over men (9:1 female-to-male ratio). ${ }^{7}$ Previous studies have examined whether gender-related pathological differences also exist in the exocrine tissues of mouse models of this disorder. ${ }^{37,38} \mathrm{MRL} / \mathrm{lpr}$ and NZB/NZW murine models develop more severe lacrimal gland inflammation in females relative to males, while male NOD mice develop worse LG inflammation than females. ${ }^{37}$ However, aged female NOD. $2_{2}{ }^{\mathrm{h} 4}$ mice develop more severe sialoadenitis when compared with age-matched male NOD. $2_{2}{ }^{\text {h }}$ mice, as assessed by histology of the salivary glands and flow cytometry of the infiltrate. ${ }^{38}$ In addition, female

$\mathrm{NOD} . \mathrm{H} 2^{\mathrm{hr}}$ mice have a significantly greater proportion of $\mathrm{CD}^{+}{ }^{+} \mathrm{T}$ cells in the infiltrate than males, with no difference in the percentage of CD8+ T Cells. ${ }^{38}$

To evaluate for any gender-related differences in the immune response of the CD25KO model, male and female CD25KO LG were histologically examined (see Supplemental Figure 1A at http://ajp.amjpathol.org). Total $\mathrm{T}$ cell infiltration was circumscribed in H\&E-stained paraffin sections at all ages and area of infiltration was calculated based on the total area of the LG. We noted that CD25KO of both genders, exhibited similar LG pathology. This was confirmed by measuring the total cell infiltration (see Supplemental Figure 1B at $h t t p: / /$ ajp.amjpathol.org).

We also performed flow cytometry analysis of CD4 and $\mathrm{CD} 8+\mathrm{T}$ cells in $\mathrm{LG}$ of CD25KO mice of both genders at ages 8,12 , and 16 weeks. We did not observe any gender difference in the phenotype of infiltrating cells (data not shown).

Taken together, these results point to a lack of gender differences in the CD25KO LG pathology.

\section{CD25KO LG Exhibit Ductal Epithelial Apoptosis Over Time}

Numerous reported studies have suggested the underlying mechanism behind glandular dysfunction in autoimmune-mediated exocrine diseases, such as SS, results from increased levels of epithelial apoptosis. ${ }^{10-13}$ Studies of the salivary glands in the NOD mouse model have demonstrated increased levels of caspase-3, one of the effector caspases essential to carrying out apoptosis, as well as an increase number of cells staining for TUNEL. ${ }^{39}$ In light of these findings, the presence of caspase-3 and TUNEL staining was evaluated in lacrimal gland specimens.

No activated (Ac)-caspase-3 staining was detected at any age in LG sections of C57BL/6 mice in the acini or epithelial ducts. In contrast, LG CD25KO mice demonstrated immunoreactivity for Ac-caspase-3 in the ductal epithelium at all ages, reaching peak intensity at 12 weeks (Figure 3A).

To confirm the results of Ac-caspase-3 staining, TUNEL staining was performed in sequential LG sections. TUNEL identifies cells that have undergone apoptosis via detection of DNA strand breaks, indicating DNA cleavage. The results of the TUNEL assay confirmed the findings of Ac-caspase-3 staining, with no TUNEL-positive cells at any age in C57BL/6 mice acini or ductal epithelial cells, while intensely positive staining was observed in the ductal epithelia of CD25KO LG at all ages (Figure 3B). It is worth noting that TUNEL immunoreactivity was absent in the infiltrating lymphocytes in the CD25KO mice (Figure 3B, dotted lines).

The absence of caspase-3 and TUNEL staining in acini of CD25KO mice could be due to a lack of acini in the regions of inflammatory cell infiltration. Taken together, these results show that CD25KO LG undergoes significantly elevated levels of ductal epithelial cell apoptosis compared with C57BL/6 wild-type mice, with sparing of the lymphocytic infiltrate. 
A
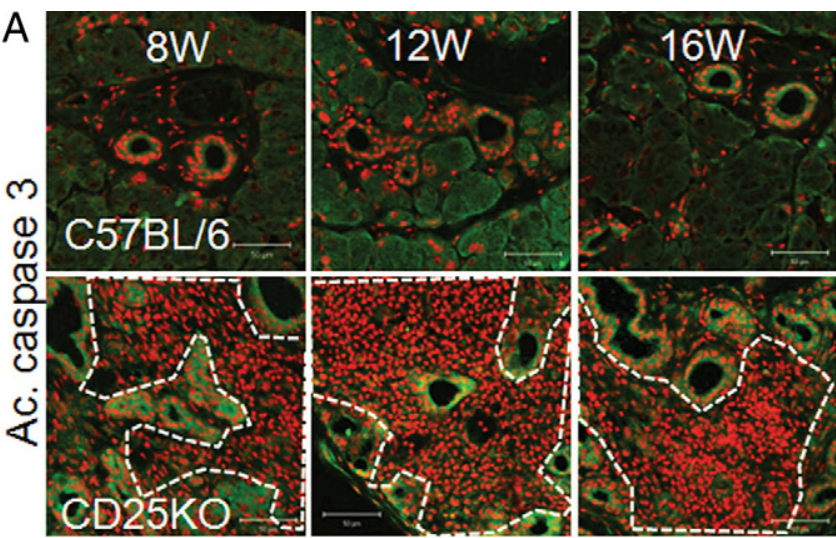

B
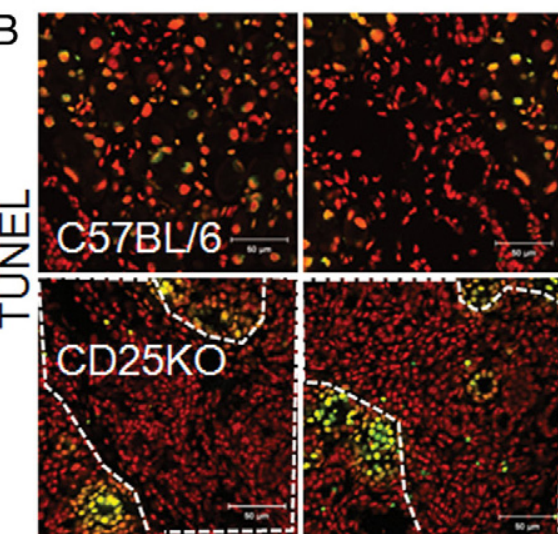

C

Peroxidase content in tears
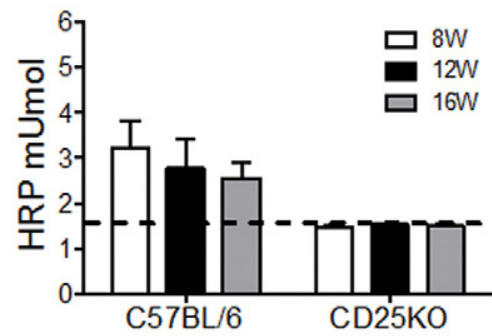

D

EGF mRNA

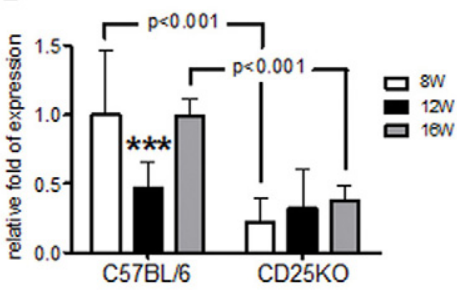

E

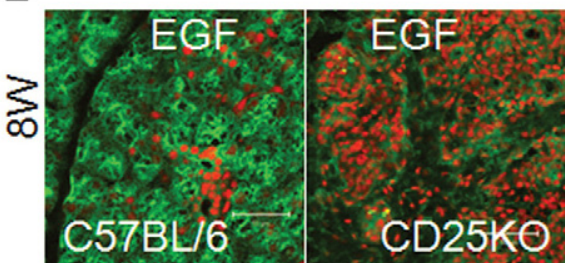

Figure 3. Merged images of laser scanning immunofluorescent confocal microscopy of lacrimal gland sections stained for activated caspase-3 (in green, A) or processed for TUNEL assay (green, B) with propidium iodide nuclear counterstaining (in red) of C57BL/6 wild-type and CD25KO mice at 8 , 12 , and 16 weeks of age. Orange color in TUNEL indicates colocalization of green and red nuclear staining. The white dotted outlines circumscribe lymphocytic infiltration of LG of $\mathrm{CD} 25 \mathrm{KO}$ mice at all ages. C: Mean \pm SD of peroxidase content in tears. Dotted lines indicate the lowest value in the linear portion of the curve generated from the observed mean fluorescent intensities versus the observed concentrations of standards. D: mRNA transcript level of EGF expression in LG lysates of both strains at all time points. E: Merged images of laser scanning immunofluorescent confocal microscopy of lacrimal gland sections stained for EGF (in green) with propidium iodide nuclear counterstaining (in red) of $\mathrm{C} 57 \mathrm{BL} / 6$ wild-type and $\mathrm{CD} 25 \mathrm{KO}$ mice at eight weeks of age. ${ }^{* * * *} P<0.001$ when compared with $\mathrm{C} 57 \mathrm{BL} / 6$ at eight weeks of age.

\section{Spontaneous Dacryoadenitis in CD25KO Mice Is Accompanied by Decreased LG Function}

To determine whether the immunopathological changes detected over time in the lacrimal glands of CD25KO and C57BL/6 mice corresponded with decreased gland secretory function, we measured tear peroxidase levels and LG epidermal growth factor (EGF) expression in both strains.

Tear peroxidase levels were measured using a spectrofluorometric assay. This method measures levels of an acinar secreted protein, peroxidase, in comparison with measuring total protein output, where other proteins not contained in secretory granules, but released on stimulation, are measured along with secretory proteins such as peroxidase. As shown in Figure 3C, peroxidase activity in tears was highest at 8 weeks in wild-type mice. By contrast, no peroxidase secretion was detected in CD25KO mice at any age. The lack of any detectable peroxidase in the knockout model suggests an inflammatory mediated secretory dysfunction of the LG.

EGF, another lacrimal gland secretory product, has been previously shown to be decreased in patients with SS, as well as other forms of dry eye disease. ${ }^{40}$ The expression of EGF was evaluated by real-time PCR in LG lysates and by immunostaining in cryosections. EGF mRNA transcripts were significantly decreased in 8 week-CD25KO LG, as compared with C57BL/6 mice of the same age (Figure 3D). Time-related changes decreased EGF mRNA transcript levels at 12 weeks in C57BL/6 mice, while EGF transcript levels remained low in CD25KO mice. Laser confocal immunomicroscopy of $L G$ at 8 weeks from both strains confirmed the low levels of expression of EGF in the CD25KO mice noted by real-time PCR. EGF immunoreactivity was noted diffusely in the acini of wild-type LG, but could not be detected detection in the CD25KO specimens (Figure 3E).

Taken together with the findings of the tear peroxidase assay, these results confirm the LG secretory impairment that takes place over time, and in the presence of autoimmune-mediated inflammation.

\section{Spontaneous Shift in T-Helper-Associated Cytokine Profile Over Time in CD25KO}

Before the discovery of the Th-17 subset, several studies in SS revealed a Th-1-predominant cytokine expression, highlighted by elevated levels of IFN- $\gamma$. Studies in NOD. IFN- $\gamma^{-1-}$ and NOD.IFN- $\gamma \mathrm{R}^{-1-}$ mice fail to undergo apo- 
ptosis and subsequently do not develop autoimmune disease of their salivary glands. ${ }^{41}$ Recent investigations focusing on the Th-17 pathway have detected an upregulation of this family in salivary glands of SS patients and NOD mice. ${ }^{26,27}$ To characterize the T-helper phenotype associated with the dacryoadenitis observed in CD25KO mice levels of mRNA transcript encoding MMP-9, Th-1-, Th-2-, and Th-17-associated genes were evaluated using real-time PCR.

Young CD25KO LG were found to contain significantly higher Th-17 (TGF- $\beta 1$, IL-17A, IL-23R, IL-21, CCL20) and Th-1-associated cytokine transcripts (IFN- $\gamma$, IL-2, IL-12, IL-12RB1, IL-18R, T-bet,) than young wild-type mice (Figure $4, A$ and $B$ ). It is worth noting that CCL2O, a potent chemoattractant molecule of Th-17-committed cells, ${ }^{14}$ displayed the greatest fold expression (400 fold) in CD25KO mice at 8 weeks of age, compared with wildtype mice. No significant changes were noted between strains in the expression of MMP-9, retinoid-related orphan receptor- $\gamma \tau$, IL-17F, IL-17Ra, IL-22, IL-6, IL-23, STAT3, IL-15, or TGF- $\beta 2$ at any age (data not shown).

A significant time-related decrease in the Th-17-related cytokine (IL-17A, IL-23R, IL-21, CCL20) mRNA transcripts was observed in CD25KO LG (Figure 4A). This was countered by an initial decrease at 12 weeks, followed by an increase at 16 weeks in the expression of multiple Th- 1 cytokines (IFN- $\gamma$, IL-2, IL-12, IL-12RB1, Tbet) in the CD25KO mice. In C57BL/6 wild-type LG, a significant time-related increase in mRNA levels of IL-2,

A
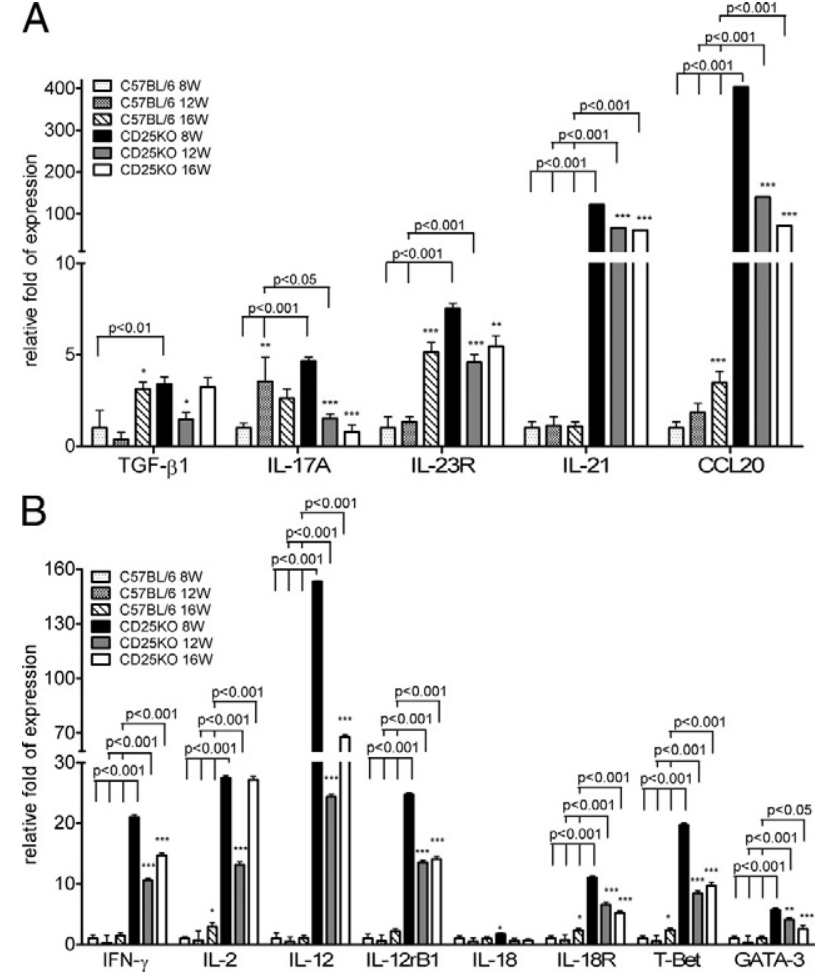

Figure 4. mRNA transcript levels of Th-17 (A) Th-1 and Th-2 (B) associated cytokines in the lacrimal glands of aged C57BL/ 6 and $\mathrm{CD} 25 \mathrm{KO}$ mice $(8,12$, and 16 weeks of age). Data are shown as the mean \pm SEM ( $n=4 /$ time point, bar indicates comparison between strains, asterisks indicate comparison within the same strain, versus 8 week, ${ }^{*} P<0.05,{ }^{* * *} P<0.01,{ }^{* * * * *} P<0.001$; $8 \mathrm{~W}=8$ weeks $12 \mathrm{~W}=12$ weeks; $16 \mathrm{~W}=16$ weeks).
IL-18R, T-bet, TGF- $\beta 1, \mathrm{IL}-23 \mathrm{R}$, and CCL20 was observed at 16 weeks, and in IL-17A at 12 weeks, with a subsequent decrease at 16 weeks (Figure 4B).

With respect to the Th-2 pathway, mRNA transcript levels of IL-4 and IL-13 were largely unchanged between both strains at all ages (data not shown). However, levels of GATA-3, the Th-2-specific transcription factor, were significantly higher in 8-week old CD25KO than C57BL/6 mice, and then proceeded to decrease at 12 and 16 weeks.

Taken together, these results suggest a time-dependent increase in the production of both Th-17 and Th-1 inflammatory mediators in C57BL/6 mice, whereas in CD25KO mice there is a switch from Th-17 to primarily Th-1 inflammation over time.

\section{Discussion}

CD25 (IL-2R $\alpha$ ) knockout mice have been proposed as an animal model of SS, due to their propensity to develop a profound lymphocytic infiltrate of multiple organs, including the colon, lung, pancreas, and bone marrow, as well as both lacrimal and salivary exocrine glands. ${ }^{30,31}$ Our results in this present study showed that CD25KO mice displayed significant lacrimal inflammation as early as 8 weeks of age, highlighted by a CD8-predominant lymphocytic infiltrate and concomitant production of proinflammatory cytokines. This inflammatory response was paralleled by ductal epithelial apoptosis, an absence of tear peroxidase, and very low EGF mRNA transcripts at all ages, reflecting the severity of gland secretory dysfunction at even a young age in these mice. Additional findings of sparse EGF immunoreactivity at 8 weeks of age in CD25KO LG compared with wild-type mice are consistent with previous studies reporting decreased EGF expression in SS patients. ${ }^{40}$ Meanwhile, our findings in C57BL/6 mice of time-related decreases in LG EGF expression suggests an impairment of $L G$ protein secretion over time, a point supported by earlier reports. ${ }^{2-4}$

It is paramount to appreciate that the previously observed changes in LG structure and function are not the same between aging and autoimmune dry eye models, despite the fact aging itself is a risk factor for dry eye. Though this study aimed to evaluate both variables, our results must be considered independently. Because of the rapid onset and severity of autoimmunity CD25KO mice develop, early mortality (12 to 16 weeks) is often a consequence; therefore, time-related changes in the CD25KO mice could only be observed up to 16 weeks. Previous studies in the aging mouse model, which normally lives up to 36 months, have implicated increased oxidative stress (marked by lipofuscin deposition) secondary to hyperglycemia and insulin resistance, as well as mast cell infiltration as causative factors for early loss of gland function. ${ }^{2-4}$ Subsequently, a lymphocyte-driven inhibition of neural function is noted to occur in much older mice..$^{2-4}$ In contrast, for autoimmune-mediated dry eye, an inflammatory cytokine-driven impairment of presynaptic and postsynaptic nerve function has been proposed to underlie glandular dysfunction early on in the 
disease process. ${ }^{42}$ This hypothesis is further supported by our observations of a $\mathrm{CD} 4^{+} \mathrm{T}$ cell-rich lymphocytic infiltrate in young CD25KO mice.

Our findings in CD25KO LGs of peaked Ac-caspase-3 and TUNEL staining at 12 and 16 weeks, respectively, partially implicate epithelial apoptosis behind decreased gland activity. Ductal epithelial cells have been shown to be important in restructuring the electrolyte composition of tears and in secreting the water component. Thus, their destruction contributes to the dysfunctional tear film that develops in lacrimal keratoconjunctivitis. These results are in agreement with multiple human ${ }^{8-10}$ and animal ${ }^{35,37}$ studies of SS and SS-like disease, which demonstrated increased levels of apoptosis-related proteins in the exocrine tissue.

Also worth mention is the overall lack of lymphocytes undergoing apoptosis noted in the present study. Indeed, reports exist documenting the relative resistance of these cells to undergoing programmed death in SS, despite their own surface expression of Fas. It has been suggested their anti-apoptotic properties derive from an overexpression of $\mathrm{Bcl}-2^{8,9}$ This expression profile gives them a propensity to replicate and survive in an otherwise destructive environment. This may also contribute to the increased risk patients with SS have of developing mucosal lymphomas. ${ }^{10}$

Both time-related changes and spontaneous autoimmune dacryoadenitis were found to be associated with increased expression of Th-1- and Th-17-related gene products, though CD25KO mice seemed to transition from a Th-17 to Th-1 bias at later time points, while the wild-type maintained a mixed composition. The T helper profile observed in CD25KO mice is comparable to previous findings in salivary gland biopsies from SS patients revealing both Th-1 and Th-17 committed cells. ${ }^{26,27}$ Given earlier reports of an IL-2-mediated Th-17 inhibition by transcriptionally suppressing its lineage factor, retinoid-related orphan receptor- $\gamma \tau^{32}$ an increase of Th-17 pathway cytokines would naturally be expected in the CD25KO mice, secondary to IL-2 inactivity. Specifically, mRNA levels of IL-17A, the signature cytokine of the Th-17 pathway, were found to be significantly higher in the LG of CD25KO mice than wild-type, with a marked time-related reduction. IL-17A expression has been identified previously in salivary gland tissue from SS patients and NOD mice. ${ }^{26,27}$ The contributions of IL-17A to autoimmunity and promoting inflammation are currently under intense investigation. Our group recently reported IL17A-promoted MMP production on the ocular surface disrupts corneal epithelial barrier function in a murine model of dry eye, and in vivo neutralization of IL-17A significantly reduces corneal MMP expression, ameliorating dessicating stress-induced barrier dysfunction. ${ }^{25} \mathrm{Ad}$ ditional studies in experimental murine dry eye have reported an IL-17A-mediated dysfunction of regulatory T cells, with a reduction of Th-17 cell expansion and restoration of Treg function after in vivo blockade of IL-17A. ${ }^{43}$

An additional Th-17-associated cytokine, IL-21, was found to have increased mRNA expression in CD25KO compared with wild-type LG, with a significant time-related decrease. An emerging role for IL-21 in autoimmu- nity is supported by observations of elevated levels of the cytokine and its receptor in the sera of patients with SS, rheumatoid arthritis, systemic sclerosis, and inflammatory bowel disease. ${ }^{44}$ Additional studies in MRL/Ipr mice modeling lupus revealed that IL-21 blockade with a soluble IL-21R-Fc fusion protein attenuated disease severity. ${ }^{45} \mathrm{~A}$ natural product of activated $\mathrm{CD}^{+}$and natural killer T cells, IL-21 has been shown to stimulate the production of pro-inflammatory molecules by epithelial cells and fibroblasts, much like IL-17A. ${ }^{12}$ However, IL-21 possesses an additional property of autocrine-mediated differentiation and amplification of mouse Th-17 cells in conjunction with TGF- $\beta 1$, and independent of IL- $6 .{ }^{12}$ In this study, levels of IL-6, the traditional Th-17 inducer, were not significantly elevated in the CD25KO LG, while $\mathrm{IL}-21$ transcripts were $>100$-fold higher than wild-type at 8 weeks. Interestingly, the IL-21 gene is located within the Idd3 locus, ${ }^{46}$ one of only two insulin-dependent diabetes (Idd) susceptibility loci (out of over 19 identified) in the NOD mouse model implicated in the simultaneous exocrinopathy these mice develop. ${ }^{47}$ Subsequently, a study of NOD spleen and pancreatic lymph nodes identified elevated expression of IL-21 and its receptor when compared with C57BL/6 controls. ${ }^{46}$

Of particular interest was the near 400-fold increase in CCL20 mRNA transcripts in the LG of young CD25KO mice, compared with C57BL/6 mice. A potent chemoattractant of Th-17 cells, CCL20 production is known to be stimulated by IL-17A itself, in addition to the pro-inflammatory cytokines IL-1 and tumor necrosis factor- $\alpha .{ }^{14}$ The significant increase in CCL20 observed in our study likely facilitated the influx of Th-17 CD4 ${ }^{+}$T cells into the LGs of CD25KO mice. Like IL-17A and IL-21, levels of CCL20 in the knockout model significantly declined with time. Taken together, this pattern of decline may be indicative of the chronic immune activation leading to a state of burnt-out inflammation and irreversible tissue injury. In accordance with this are the previously noted gross and histological findings of small, atrophic glands and massive fibrotic replacement of acinar tissue in the 16-week old mice as compared with the red, inflamed glands of the 8- and 12-week old CD25KO mice.

In addition to elevated Th-17 expression, we detected significantly increased levels of the Th- 1 signature cytokine, IFN- $\gamma$, in the CD25KO mice, with peaks at 8 and 16 weeks. Increased production of IFN- $\gamma$ in patients with SS has been well documented in the literature, with numerous studies detecting its overexpression in lacrimal and salivary glands. ${ }^{27}$ Both NOD.IFN- $\gamma^{-1-}$ and NOD.IFN$\gamma \mathrm{R}^{-1-}$ mice failed to undergo lymphocytic infiltration of the salivary gland, and retained normal secretory function. ${ }^{41}$ Increased levels of IFN- $\gamma$ have also been found to up-regulate epithelial expression of Fas and caspase-8, thus potentiating the apoptotic pathway. ${ }^{8}$ Additionally, a recent study characterizing a transgenic mouse model of SS overexpressing IL-12, the chief stimulator of the Th-1 axis and subsequent IFN- $\gamma$ production, detected focal lymphocytic infiltration of the salivary glands, $L G$, and the lungs, mimicking a SS phenotype. ${ }^{48}$ Levels of IL-12, its 
receptor, IL-RB1, and the Th-1 lineage specific transcription factor, T-bet, were all significantly increased in our animal model of SS when compared with C57BL/6 mice.

Another Th-1-related cytokine, IL-2, was found to be significantly higher in CD25KO than C57BL/6 mice. Though it has been shown to drive differentiation of naïve T cells toward Th- 1 commitment, increased levels in this study could be due to a compensatory feedback mechanism from impaired IL-2 signaling at the receptor level. Normally, IL-2 signals through its high affinity receptor complex, IL-2R $\alpha \beta \gamma$, to mediate the development and maintenance of CD4+CD25+Foxp3+ regulatory T cells, which are crucial in preventing organ-specific autoimmunity. ${ }^{28,29}$ In humans, mutation of Foxp3 results in the lethal autoimmune disorder, immune dysregulation, polyendocrinopathy, enteropathy, x-linked syndrome (IPEX), characterized by a congenital defect in Treg expression. ${ }^{49}$ Similarly, Foxp3 mutant scurfy mice develop multiorgan autoimmunity and die at a young age..$^{30,49}$ The importance of Tregs in suppressing ocular surface inflammation has been demonstrated by reconstituting Tcell-deficient nude mice with either pathogenic $\mathrm{CD} 4^{+} \mathrm{T}$ cells from donors exposed to dessicating stress alone, or in combination with in vitro expanded CD4+CD25+ Foxp3+ regulatory $T$ cells. ${ }^{50}$ Nude mice reconstituted with both pathogenic $\mathrm{CD} 4^{+} \mathrm{T}$ cells and in vitro Tregs had significantly lower conjunctival cellular infiltration and tear levels of pro-inflammatory cytokines compared with the mice receiving only pathogenic effector T cells. ${ }^{50}$ Other than dry eye, defective Treg function has been linked to the pathogenesis of several autoimmune diseases including multiple sclerosis and rheumatoid arthritis.

Altogether, it has become increasingly evident that a Th-1/Th-17 dichotomy is not so absolute, and there may be unique contributions of each pathway to the total makeup of autoimmunity. Notably, a murine model of experimental autoimmune encephalomyelitis where IFN$\gamma$-producing Th- 1 cells facilitated the entry of Th- 17 cells into the central nervous system, illustrates the collaboration between the two pathways. ${ }^{51} \mathrm{Th}-17$ preparations that lacked IFN- $\gamma$-producing cells were unable to cause disease, leading the investigators to conclude that only Th-1 cells can access a noninflamed central nervous system. ${ }^{51}$ Though speculative, the possibility of Th-17 cells not being terminally differentiated and retaining the plasticity to develop into IFN- $\gamma$ secreting Th- 1 cells with age or under some unknown stimulus is intriguing, especially given recent reports of T cells known as "double producers" that simultaneously express IFN- $\gamma$ and IL-17.52

In summary, we demonstrated that the autoimmune exocrinopathy of the CD25KO LG is composed of a dynamic Th-1 and Th-17 response, with a Th-17 phenotype in young mice shifting to a Th-1-predominant expression at later time points. In addition, we provided evidence for a role of both ductal epithelial apoptosis, as well as defective effector T cell apoptosis, in the LG dysfunction accompanying the inflammatory infiltrate. The results of this study should yield further insight into the complex pathogenesis of Sjögren's syndrome.

\section{References}

1. Moss SE, Klein R, Klein BE: Incidence of dry eye in an older population. Arch Ophthalm 2004, 122:369-373

2. Obata $\mathrm{H}$ : Anatomy and histopathology of the human lacrimal gland Cornea 2006, 25:S82-S89

3. Rios JD, Horikawa Y, Chen LL, Kublin CL, Hodges RR, Dartt DA, Zoukhri D: Age-dependent alterations in mouse exorbital lacrima gland structure, innervation, and secretory response. Exp Eye Research 2005, 80:477-491

4. Rocha EM, Alves M, Rios JD, Dartt DA: The aging lacrimal gland: changes in structure and function. Ocul Surf 2008, 6:162-174

5. Miljanovic B, Dana R, Sullivan DA, Schaumberg DA: Impact of dry eye syndrome on vision-related quality of life. Am J Ophthalmol 2007, 143:409-415

6. Chotikavanich S, De Paiva CS, Li de Q, Chen JJ, Bian F, Farley WJ, Pflugfelder SC: Production and activity of matrix metalloproteinase-9 on the ocular surface increase in dysfunctional tear syndrome. Invest Ophthalmol Vis Sci 2009, 50:3203-3209

7. Fox RI, Kang HI: Pathogenesis of Sjögren's syndrome. Rheum Dis Clin North Am 1992, 18:517-538

8. Kong L, Ogawa N, Nakabayashi T, Liu GT, D'Souza E, McGuff HS Guerrero D, Talal N, Dang H: Fas and Fas ligand expression in the salivary glands of patients with primary Sjögren syndrome. Arthritis Rheum 1997, 40:87-97

9. Kong L, Ogawa N, McGuff HS, Nakabayashi T, Sakata KM, Masago $\mathrm{R}$, Vela-Roch $\mathrm{N}$, Talal N, Dang $\mathrm{H}$ : Bcl-2 family expression in salivary glands from patients with primary Sjögren's syndrome: involvement of Bax in salivary gland destruction. Clin Immunol Immunopathol 1998, 88:133-141

10. Manganelli P, Fietta P: Apoptosis and Sjögren syndrome. Seminars in Arthritis and Rheumatism 2003, 33:49-65

11. Vieldhoen M, Hocking RJ, Atkins CJ, Locksley RM, Stockinger B: TGF-beta in the context of an inflammatory cytokine milieu supports de novo differentiation of IL-17 producing T cells. Immunity 2006 , 24:179-189

12. Korn T, Bettelli E, Gao W, Awasthi A, Jager A, Strom TB, Oukka M, Kuchroo VK: IL-21 initiates an alternative pathway to induce proinflammatory $\mathrm{T}(\mathrm{H}) 17$ cells. Nature 2007, 448:484-487

13. Zhou L, Lopes JE, Chong MM, Ivanov II, Min R, Victora GD, Shen Y, Du J, Rubtsov YP, Rudensky AY, Ziegler SF, Littman DR: TGF-betainduced Foxp3 inhibits $\mathrm{T}(\mathrm{H}) 17$ cell differentiation by antagonizing RORgammat function. Nature 2008, 453:236-240

14. Homey B, Dieu-Nosjean MC, Wiesenborn A, Massacrier C, Pin JJ, Oldham E, Catron D, Buchanan ME, Muller A, DeWaal Malefyt R, Deng G, Orozco R, Ruzicka T, Lehmann P, Lebecque S, Caux C, Zlotnik A: Up-regulation of macrophage inflammatory protein-3 alpha/ CCL20 and CC chemokine receptor 6 in psoriasis. J Immunol 2000, 164:6621-6632

15. Liang SC, Tan XY, Luxenberg DP, Dunussi-Joannopoulos K, Collins M, Fouser LA: Interleukin (IL)-22 and IL-17 are coexpressed by Th17 cells and cooperatively enhance expression of anatimicrobial peptides. J Exp Med 2006, 203:2271-2279

16. Cortez DM, Feldman MD, Mummidi S, Valente AJ, Steffensen B, Vincenti M, Barnes JL, Chandrasekar B: IL-17 stimulates MMP-1 expression in primary human cardiac fibroblasts via p38. Am J Physiol Heart Circ Physiol 2007, 293:H3356-H3365

17. Yagi $Y$, Andoh A, Inatomi $O$, Tsujikawa $T$, Fujiyama $Y$ : Inflammatory responses induced by interleukin-17 family members in human coIonic subepithelial myofibroblasts. J Gastroenterol 2007, 42:746-753

18. Komiyama $Y$, Nakae S, Matsuki T, Nambu A, Ishigame H, Kakuta S, Sudo K, Iwakura Y: IL-17 plays an important role in the development of experimental autoimmune encephalomyelitis. J Immunol 2006, 117:566-573

19. Nakae S, Komiyama Y, Nambu A, Sudo K, Iwase M, Homma I, Sekikawa K, Asano M, Iwakura Y: Antigen-specific T cell sensitization is impaired in IL-17-deficient mice, causing suppression of allergic cellular and humoral responses. Immunity 2002, 17:375-387

20. Nakae S, Nambu A, Sudo K, Iwakura A: Suppression of immune induction of collagen-induced arthritis in IL-17 deficient mice. J Immunol 2003, 171:6173-6177

21. Hwang SY, Kim HY: Expression of IL-17 homologs and their receptors in the synovial cells of rheumatoid arthritis patients. Mol Cells 2005, 19:180-184 
22. Wong CK, Ho CY, Li EK, Lam CW: Elevation of proinflammatory cytokine (IL-18, IL-17, IL-12) and Th2 cytokine (IL-4) concentrations in patients with systemic lupus erythematosus. Lupus 2000, 9:589-593

23. Tzartos JS, Friese MA, Craner MJ, Palace J, Newcombe J, Esiri MM, Fugger L: Interleukin-17 production in central nervous system-infiltrating $\mathrm{T}$ cells and glial cells is associated with activate disease in multiple sclerosis. Am J Path 2008, 172:146-155

24. Chi W, Zhu X, Yang $P$, Liu X, Lin X, Zhou H, Huang X, Kijlstra A: Upregulated IL-23 and IL-17 in Behcet patients with active uveitis. Invest Ophthalm Vis Sci 2008, 49:3058-3064

25. De Paiva CS, Chotikavanich S, Pangelinan SB, Pitcher JD 3rd, Fang B, Zheng X, Ma P, Farley WJ, Seimasko KF, Niederkorn JY, Stern ME, Li DQ, Pflugfelder SC: IL-17 disrupts corneal barrier following desiccating stress. Mucosal Immunology 2009, 2:243-253

26. Nguyen CQ, Hu MH, Li Y, Stewart C, Peck AB: Salivary gland tissue expression of interleukin-23 and interleukin-17 in Sjogren's syndrome: findings in humans and mice. Arthritis Rheum 2008, 58:734-743

27. Sakai A, Sugawara Y, Kuroishi T, Sasano T, Sugawara S: Identification of IL-18 and Th17 cells in salivary glands of patients with Sjogren's syndrome, and amplification of IL-17-mediated secretion of inflammatory cytokines from salivary gland cells by IL-18. J Immunol 2008, 181:2898-2906

28. Fontenot JD, Gavin MA, Rudensky AY: Foxp3 programs the development and function of CD4+CD25+ regulatory T cells. Nat Immunol 2003, 4:330-336

29. Kim JM, Rasmussen JP, Rudensky AY: Regulatory T cells prevent catastrophic autoimmunity throughout the lifespan of mice. Nat Immunol 2007, 8:191-197

30. Sharma R, Zheng L, Guo X, Fu SM, Ju ST, Jarjour WN: Novel animal models for Sjögren's syndrome: expression and transfer of salivary gland dysfunction from regulatory $T$ cell-deficient mice. J Autoimmun 2006, 4:289-296

31. Sadlack B, Merz H, Schorle H, Schimpl A, Feller AC, Horak I: Ulcerative colitis-like disease in mice with a disrupted interleukin 2 gene. Cell 1993, 75:253-261

32. Laurence A, Tato CM, Davidson TS, Kanno Y, Chen Z, Yao Z, Blank RB, Meylan F, Siegel R, Hennighausen L, Shevach Em, O'Shea JJ: Interleukin-2 signaling via STAT5 constrains T helper 17 cell generation. Immunity 2007, 26:371-381

33. Marcozzi G, Liberati V, Madia F, Centofanti M, de Feo G: Age- and gender-related differences in human lacrimal fluid peroxidase activity. Ophthalmologica 2003, 217:294-297

34. De Paiva CS, Corrales RM, Villarreal AL, Farley WJ, Li DQ, Stern ME, Pflugfelder SC: Apical corneal barrier disruption in experimental murine dry eye is abrogated by methylprednisolone and doxycycline. Invest Ophthalmol Vis Sci 2006, 47:2847-2856

35. Yeh S, Song XJ, Farley W, Li DQ, Stern ME, Pflugfelder SC: Apoptosis of ocular surface cells in experimentally induced dry eye. Invest Ophthalmol Vis Sci 2003, 44:124-129

36. De Paiva CS, Hwang CS, Pitcher JD 3rd, Pangelinan SB, Rahimy E, Chen W, Yoon KC, Farley WJ, Niederkorn JY, Stern ME, Li DQ, Pflugfelder SC: Age-related T-cell cytokine profile parallels corneal disease severity in Sjogren's syndrome-like keratoconjunctivitis sicca in CD25KO mice. Rheumatology 2010, 49:246-258

37. Toda I, Sullivan BD, Rocha EM, Da Silveira LA, Wickham LA, Sullivan DA: Impact of gender on exocrine gland inflammation in mouse models of Sjogren's syndrome. Exp Eye Res 1999, 69:355-366

38. Ciháková D, Talor MV, Barin JG, Baldeviano GC, Fairweather D, Rose
NR, Burek CL: Sex differences in a murine model of Sjogren's syndrome. Ann NY Acad Sci 2009, 1173:378-383

39. Masago R, Aiba-Masago S, Talal N, Zuluaga FJ, Al-Hashimi I, Moody M, Lau CA, Peck AB, Brayer J, Humphreys-Beher MG, Dang $\mathrm{H}$ : Elevated levels of bax and caspase-3 activation in the NOD-scid model of Sjögren syndrome. Arthritis Rheum 2001, 44:693-702

40. Pflugfelder SC, Jones D, Ji Z, Afonso A, Monroy D: Altered cytokine balance in the tear fluid and conjunctiva of patients with Sjögren's syndrome keratoconjunctivitis sicca. Curr Eye Res 1999, 19:201-211

41. Cha S, Brayer J, Gao J, Brown V, Killedar S, Yasunari U, Peck AB: A dual role for interferon- $\gamma$ in the pathogenesis of Sjögren's syndromelike autoimmune exocrinopathy in the nonobese diabetic mouse. Scand J Immunol 2004, 60:552-565

42. Zoukhri D, Kublin CL: Impaired neurotransmitter release from lacrimal and salivary gland nerves of a murine model of Sjögren's syndrome. Invest Ophthalmol Vis Sci 2001, 42:925-932

43. Chauhan SK, El Annan J, Ecoiffier T, Goyal S, Zhang Q, Saban DR, Dana R: Autoimmunity in dry eye is due to resistance of Th17 to Treg suppression. J Immunol 2009, 182:1247-1252

44. Yuan SL, Jiang L, Zhang XL, Li SF, Duan HM, Wang XF: Serum IL-21 level in patients with primary Sjogren's syndrome and clinical significance of IL-21. Xi Bao Yu Fen Zi Mian Yi Xue Za Zhi 2007, 23:124-126

45. Herber D, Brown TP, Liang S, Young DA, Collins M, Dunussi-Joannopoulos K: IL-21 has a pathogenic role in a lupus-prone mouse model and its blockade with IL-21R. Fc reduces disease progression. J Immunol 2007, 178:3822-3830

46. King C, Ilic A, Koelsch K, Sarvetnick N: Homeostatic expansion of T cells during immune insufficiency generates autoimmunity. Cell 2004, 117:265-277

47. Brayer J, Lowry J, Cha S, Robinson CP, Yamachika S, Peck AB Humphreys-Beher MG: Alleles from chromosomes 1 and 3 of NOD mice combine to influence Sjögren's syndrome-like autoimmune exocrinopathy. J Rheumatol 2000, 27:1896-1904

48. McGrath-Morrow S, Laube B, Tzou SC, Cho C, Cleary J, Kimura H, Rose NR, Caturegli P: IL-12 overexpression in mice as a model for Sjögren lung disease. Am J Physiol Lung Cell Mol Physiol 2006, 291:L837-L846

49. Wildin RS, Ramsdell F, Peake J, Faravelli F, Casanova JL, Buist N, Levy-Lahad E, Mazzella M, Goulet O, Perroni L, Bricarelli FD, Byrne G, McEuen M, Proll S, Appleby M, Brunkow ME: X-linked neonatal diabetes mellitus, enteropathy, and endocrinopathy syndrome is the human equivalent of mouse scurfy. Nat Genet 2001, 27:18-20

50. Siemasko KF, Gao J, Calder VL, Hanna R, Calonge M, Pflugfelder SC, Niederkorn JY, Stern ME: In vitro expanded CD4+CD25+Foxp3+ regulatory $T$ cells maintain a normal phenotype and suppress immune-mediated ocular surface inflammation. Invest Ophthalmol Vis Sci 2008, 49:5434-5440

51. O'Connor RA, Prendergast CT, Sabatos CA, Lau CW, Leech MD Wraith DC, Anderton SM: Cutting edge; Th1 cells facilitate the entry of Th17 cells to the central nervous system during experimental autoimmune encephalomyelitis. J Immunol 2008, 181:3750-3754

52. Eid RE, Rao DA, Zhou J, Lo SL, Ranjbaran H, Gallo A, Sokol SI, Pfau S, Pober JS, Tellides G: Interleukin-17 and interferon- $\gamma$ are produced concomitantly by human coronary artery-infiltrating t cells and act synergistically on vascular smooth muscle cells. Circulation 2009, 119:1424-1432 